\title{
Acute Reversible Heart Failure Caused by Coronary Vasoconstriction due to Continuous 5-Fluorouracil Combination Chemotherapy
}

\author{
Cornelia Dechant $^{a} \quad$ Martina Baur ${ }^{b}$ Rudolf Böck ${ }^{a}$ \\ Martin Czejka ${ }^{c}$ Andrea Podczeck-Schweighofer ${ }^{a}$ \\ Christian Dittrich $^{\mathrm{b}}$ Günter Christ ${ }^{\mathrm{a}}$ \\ ${ }^{a}$ Department of Cardiology and ${ }^{b}$ LBI-ACR VIEnna and ACR-ITR VIEnna Centre for \\ Oncology and Haematology, Kaiser Franz Josef Hospital, and ${ }^{\mathrm{C} D e p a r t m e n t}$ of \\ Clinical Pharmacy and Diagnostics, University of Vienna, Vienna, Austria
}

\section{Key Words}

5-Fluorouracil · Coronary vasoconstriction · Acute heart failure

\begin{abstract}
We present the case of a 51-year-old male patient who received adjuvant chemotherapy consisting of oxaliplatin, bolus and continuous 5-fluorouracil (5-FU) and leucovorin after anterior resection because of locally advanced rectal cancer. Preoperative chemotherapy with capecitabine (an oral 5-FU prodrug) had been well tolerated. Two days after initiation of the first course of chemotherapy, the patient reported typical chest pain. The ECG showed ST elevations and prominent T waves in almost all leads. Due to suspicion of a high-risk acute coronary syndrome, an urgent cardiac catheterization was performed. It showed a generally reduced coronary flow with multiple significant stenoses (including the ostia of the left and right coronary artery), as well as a highly reduced left ventricular function with diffuse hypokinesia. Due to the meanwhile completely stable situation of the patient after medical acute coronary syndrome treatment, no ad hoc intervention was performed to allow further discussion of the optimal management. Thereafter, the patient remained clinically asymptomatic, without any rise in cardiac necrosis parameters; only NT-pro-BNP was significantly elevated. A control cardiac catheterization 2 days later revealed a restored normal coronary artery flow with only coronary calcifications without significant stenoses, as well as a normal left ventricular ejection fraction. Cardiovascular symptoms occurred on the second day of continuous 5-FU treatment. As cardiotoxic effects seem to appear more frequently under continuous application of 5-FU, compared to the earlier established 5-FU bolus regimens, treating medical oncologists should pay special attention to occurring
\end{abstract}


cardiac symptoms and immediately interrupt 5-FU chemotherapy and start a cardiologic work-up.

\section{Introduction}

The cytostatic drug 5-fluorouracil (5-FU) is used for the treatment of malignant tumors of the gastrointestinal tract, the head and neck region as well as breast cancers. For development of 5-FU cardiotoxicity, several different causative mechanisms like occurrence of coronary artery spasm [1], direct non-ischemic toxic effects on myocardial cells [2], endothelial damage [3], or decreased oxygen concentrations in the blood [4] are considered. Cardiotoxic effects are also described for capecitabine, an oral 5-FU prodrug [5].

We report a case of acute transient heart failure caused by 5-FU-induced general coronary vasoconstriction while the patient was still under continuous infusion of 5-FU in combination with leucovorin and oxaliplatin during the second day of administration of the drug.

\section{Case Report}

A 51-year-old male patient with locally advanced rectal cancer and status post-anterior resection of the rectum and post-preoperative chemoradiation with capecitabine postoperatively received adjuvant combination chemotherapy consisting of oxaliplatin $85 \mathrm{mg} / \mathrm{m}^{2}$, leucovorin $400 \mathrm{mg} / \mathrm{m}^{2}$, and 5 -FU ( $400 \mathrm{mg} / \mathrm{m}^{2}$ bolus i.v. followed by $2,400 \mathrm{mg} / \mathrm{m}^{2}$ continuous infusion over $48 \mathrm{~h}$ i.v.) corresponding to modified FOLFOX 6 schedule on an outpatient basis.

In his medical history, no cardiac events had been reported, but a high cardiovascular risk profile was present with arterial hypertension, hyperlipidemia, current cigarette smoking (80 pack-years), and known peripheral artery disease. On the second day of the continuous 5-FU infusion, the patient reported typical chest pain. The ECG ( $\underline{\text { fig. 1) }}$ ) showed significant ST elevations and prominent T waves in almost all leads (I-III, aVF, and $V_{2}-V_{6}$ ); blood pressure was normal with $130 / 70 \mathrm{~mm} \mathrm{Hg}$. After starting acute coronary syndrome therapy (including platelet function inhibition with prasugrel and acetylsalicylic acid), the ECG returned to normal and the clinical symptoms improved. However, due to the significant dynamic ST changes, an acute coronary angiography was performed. It revealed a generally reduced coronary flow (TIMI II-III), with an ostial stenosis of the left main coronary artery, multiple focal stenoses in the circumflex artery, the left anterior descending coronary artery and the diagonal branch (fig. 2a), and an ostial stenosis of the right coronary artery. Left ventricular ejection fraction (LVEF) was highly reduced (24\%) with diffuse hypokinesia (fig. 2b) and a highly elevated left ventricular end-diastolic filling pressure (LVEDP) of $30 \mathrm{~mm} \mathrm{Hg}$. Due to the already normalized ECG and since the patient was meanwhile asymptomatic, no ad hoc intervention was performed to allow further interdisciplinary discussion of optimal further management. During the following days, cardiac necrosis parameters remained negative, only NT-pro-BNP plasma level was significantly elevated $(2,960 \mathrm{pg} / \mathrm{ml})$. A medical therapy with an ACE inhibitor, diuretics, and a calcium channel blocker was established. Echocardiographic follow-up 2 days later surprisingly showed a marked improvement of left ventricular function (LVEF approximately 50-55\%). Follow-up angiography 2 days after first angiography showed a normal coronary flow (TIMI III) and coronary calcifications without significant stenoses (fig. 3a). LVEF with 60\% was also normal (fig. 3b) and LVEDP with 16 $\mathrm{mm} \mathrm{Hg}$ was only marginally elevated. The patient could be discharged in good clinical condition with oral medication including acetylsalicylic acid, verapamil, and atorvastatin. At follow-up after 5 months, a normal NT-pro-BNP was recorded $(96.95 \mathrm{pg} / \mathrm{ml})$, and the patient remained free of further cardiac symptoms. 


\section{Discussion}

To our knowledge, this case report represents the first detailed documentation of the potentially life-threatening complication of generalized coronary vasoconstriction in combination with acute transient heart failure and its reversibility in connection with adjuvant chemotherapy consisting of 5-FU (bolus and continuous), leucovorin, and oxaliplatin.

Concerning the 5-FU plasma levels achieved while administering the bolus and continuous 5-FU schedule (also called FOLFOX schedule), 5-FU steady-state plasma levels on the first and second day of treatment of about $340 \mathrm{ng} / \mathrm{ml}$ are observed [6]. These 5-FU plasma levels correspond to the 5-FU plasma levels of $\sim 30 \mathrm{ng} / \mathrm{ml}$ and 900 $\mathrm{ng} / \mathrm{ml}$, respectively, reached when capecitabine is delivered at $1,250 \mathrm{mg} / \mathrm{m}^{2}[7,8]$ and at $825 \mathrm{mg} / \mathrm{m}^{2}$ [9], the meanwhile established standard for chemoradiation [10], which was used in the case presented. 5-FU maximal plasma levels reached by bolus injections exceed by 100 -fold the maximal concentration of 5-FU metabolized from capecitabine or continuous 5-FU infusions plus leucovorin [6]. Therefore, presumably, maximal peak concentrations of 5-FU levels do not play the key role in producing 5 -FUtriggered toxicity.

According to Kosmas et al. [11], 5-FU toxicity is a schedule-dependent event, resulting in higher toxicity of $6.7 \%$ on continuous infusion compared to short-term 5FU infusion (2.3\%). In addition to continuous 5-FU, the application of leucovorin contributes to increased cardiotoxicity [11].

Theoretically, administration of oxaliplatin which preceded administration of 5-FU might have had an independent or an additional effect on the coronary vessels. Although a pharmacologic drug interaction between oxaliplatin and 5-FU may not be completely excluded, the occurrence of a pharmacokinetic drug-drug interaction seems unlikely [12].

It is noteworthy that our patient tolerated oral intake of capecitabine, an oral 5-FU prodrug, for 5 weeks during preoperative chemoradiation without occurrence of clinically symptomatic coronary vasoconstriction. In our opinion, this phenomenon remains unexplained and is in contrast with the observation made by Shaib et al. [13]. They reported a patient with repeated cardiotoxic symptoms following administration of either capecitabine or continuous 5-FU in form of the FOLFOX (5-FU, oxaliplatin, and leucovorin) regimen but not following bolus 5-FU intravenously, as used in the FLOX regimen (5-FU, leucovorin, and oxaliplatin) [13]. To speculate, individual disposition, environmental influences, or medication interactions may have played a role in our patient who developed cardiac toxicity on exposition to modified FOLFOX 6 schedule but not on capecitabine. However, once patients have experienced cardiac toxicity caused by 5-FU, they should not be rechallenged as the risk of recurrence of cardiotoxicity is known to be high [14].

In summary, our case report shows that 5-FU infusion (bolus and continuous) combined with leucovorin and oxaliplatin is able to induce coronary vasoconstriction leading to acute but reversible heart failure. Cardiovascular symptoms due to 5-FU toxicity occurred typically on day 2 of the first cycle of chemotherapy [15]. Cardiotoxic events caused by continuous application of 5-FU seem to appear more frequently 
compared to the historically administered 5-FU bolus regimens. In case cardiac symptoms occur, the treating medical oncologists should immediately interrupt the 5FU-containing (poly)chemotherapy and start a cardiologic work-up. Rechallenge with 5-FU is not recommended. Pursuing this strategy, reversion of cardiotoxicity is possible as shown in our case. Previous symptomless tolerance of capecitabine does not preclude occurrence of toxicity by 5-FU (bolus and continuous) administration in combination with leucovorin and oxaliplatin.

\section{Disclosure Statement}

The authors have no conflicts of interest.

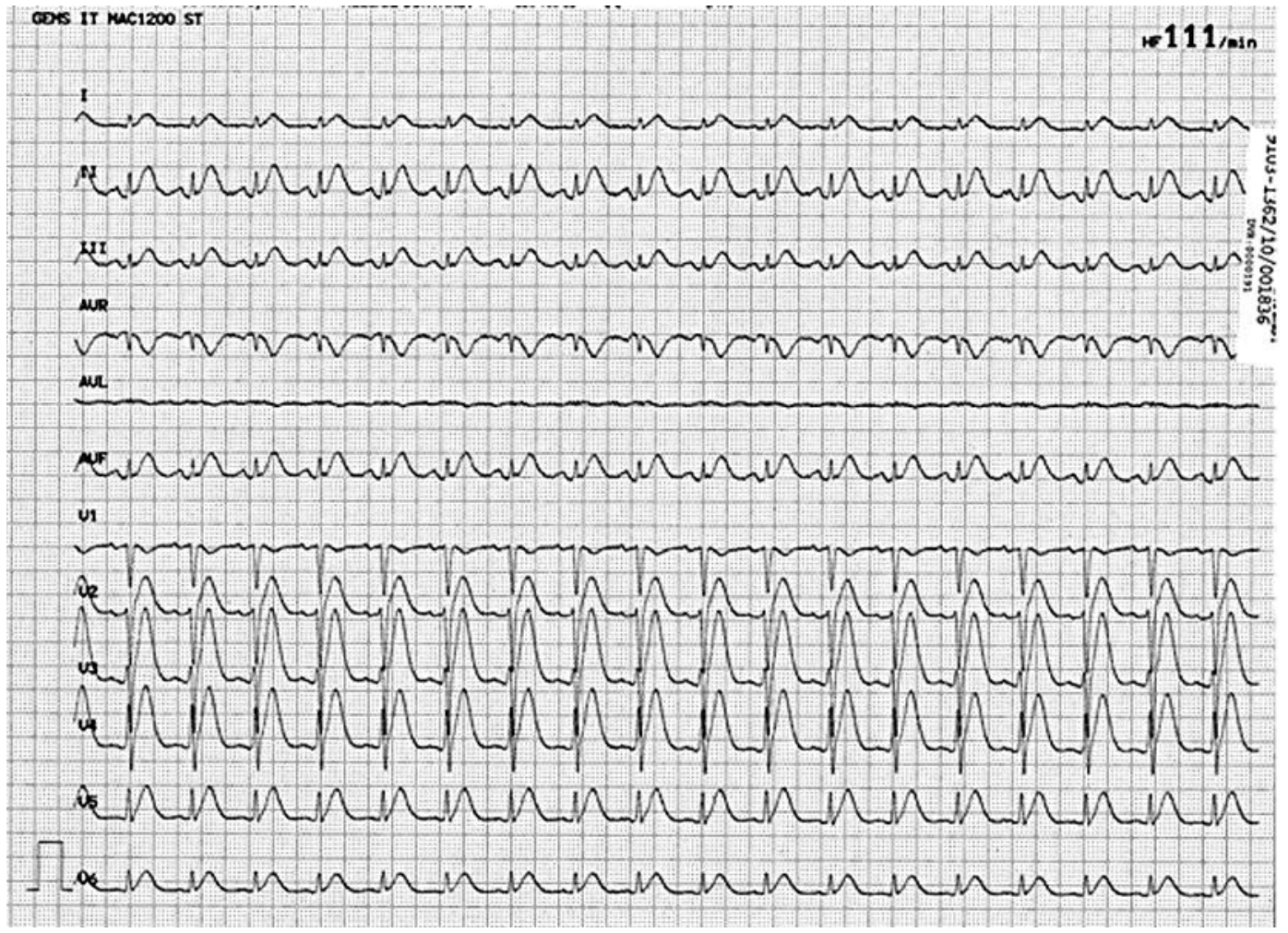

Fig. 1. ECG with ST elevations and prominent $T$ waves (I-III, aVF, and in $V_{2}-V_{6}$ ). 

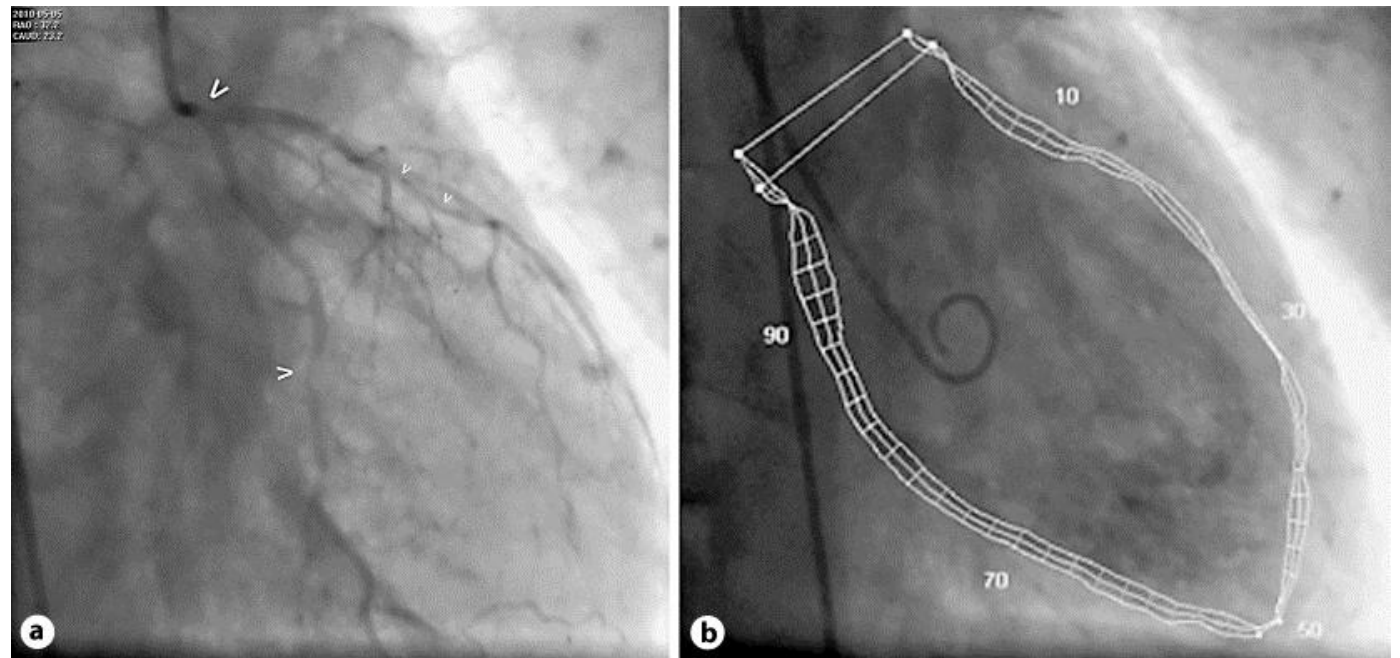

Fig. 2. Acute cardiac catheterization. a Caudal RAO projection of the left coronary artery, with ostial left main stenosis (bold arrow), mid circumflex artery stenosis (normal arrow), and diffuse calcified left anterior descending artery stenosis (small arrows). b Ventriculography (RAO $30^{\circ}$ ). Quantitative evaluation revealed a highly reduced left ventricular function (LVEF 24\%) with diffuse hypokinesia and highly elevated LVEDP of $30 \mathrm{~mm} \mathrm{Hg}$.
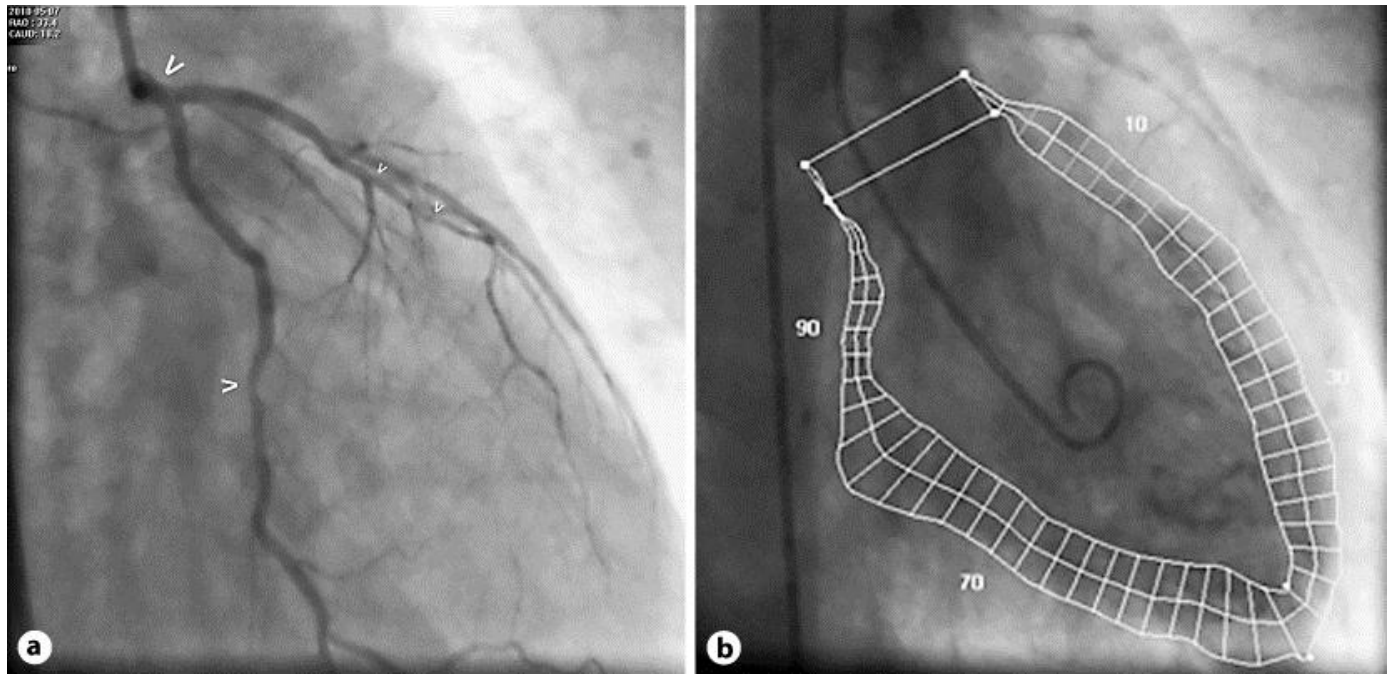

Fig. 3. Control cardiac catheterization 2 days later. a Caudal RAO projection of the left coronary artery: normalized left main (bold arrow) and non-significant circumflex artery stenosis (normal arrow), diffusely non-significantly diseased left anterior descending artery (small arrows). b Ventriculography $\left(\right.$ RAO $30^{\circ}$ ). Quantitative evaluation revealed a normal left ventricular function (LVEF 60\%) and no wall motion abnormalities, with near normal LVEDP of $16 \mathrm{~mm} \mathrm{Hg}$. 


\section{References}

$\checkmark 1$ Luwaert RJ, Descamps O, Majois F, Chaudron JM, Beauduin M: Coronary artery spasm induced by 5 fluorouracil. Eur Heart J 1991;12:468-470.

2 Chaudary S, Thomas Song SY, Jaski BE: Profound, yet reversible, heart failure secondary to 5fluorouracil. Am J Med 1988;85:454-456.

-3 Cwikiel M, Eskilsson J, Albertsson M, Stavenow L: The influence of 5 -fluorouracil and methotrexate on vascular endothelium. Ann Oncol 1996;7:731-737.

4 Spasojević I, Jelić S, Zakrzewska J, Bačić G: Decreased oxygen transfer capacity of erythrocytes as a cause of 5-fluorouracil related ischemia. Molecules 2009;14:53-56.

5 Coughlin S, Das S, Lee J, Cooper J: Capecitabine induced vasospastic angina. Int J Cardiol 2008;130:e34e36.

-6 Joulia JM, Pinguet F, Ychou M, Duffour J, Topart D, Grosse PY, Astre C, Bressolle F: Pharmacokinetics of 5 fluorouracil (5-FUra) in patients with metastatic colorectal cancer receiving 5-FUra bolus plus continuous infusion with high dose folinic acid (LV5FU2). Anticancer Res 1997;17:2727-2730.

7 Schüller J, Cassidy J, Dumont E, Roos B, Durston S, Banken L, Utoh M, Mori K, Weidekamm E, Reigner B: Preferential activation of capecitabine in tumor following oral administration to colorectal cancer patients. Cancer Chemother Pharmacol 2000;45:291-297.

-8 Cassidy J, Dirix L, Bissett D, Reigner B, Griffin T, Allman D, Osterwalder B, Van Osterom AT: A phase I study of capecitabine in combination with oral leucovorin in patients with intractable solid tumors. Clin Cancer Res 1998;4:2755-2761.

-9 Chang DZ, Olencki T, Budd GT, Peereboom D, Ganapathi R, Osterwalder B, Bukowski R: Phase I trial of capecitabine in combination with interferon alpha in patients with metastatic rectal cancer: toxicity and pharmacokinetics. Cancer Chemother Pharmacol 2001;48:493-498.

-10 Dunst J, Debus J, Rudat V, Wulf J, Budach W, Hoelscher T, Reese T, Mose S, Roedel C, Zuehlke H, Hinke A: Neoadjuvant capecitabine combined with standard radiotherapy in patients with locally advanced rectal cancer. Strahlenther Onkol 2008;184:450-456.

11 Kosmas C, Kallistratos MS, Kopterides P, Syrios J, Skopelitis H, Mylonakis N, Karabelis A, Tsavaris N: Cardiotoxicity of fluoropyrimidines in different schedules of administration: a prospective study. J Cancer Res Clin Oncol 2008;134:75-82.

12 Farkouh A, Schueller J, Scheithauer W, Czejka M: Plasma disposition of capecitabine (CCB) and its metabolites 5'-deoxy-5-fluorocytidine (5'-DFCR) and 5'-deoxy-5-fluorouracil (5'-DFUR) with two different capecitabine/oxaliplatin dosage regimens. Int J Clin Pharmacol Ther 2010;48:487-488.

$\checkmark 13$ Shaib W, Lee V, Saif MW: Bolus 5-fluorouracil as an alternative modality to infusion 5-fluorouracil in a patient with rectal cancer and capecitabine-induced cardiotoxicity. In Vivo 2009;23:821-826.

$\$ 14$ Ng M, Cunningham D, Norman AR: The frequency and pattern of cardiotoxicity observed with capecitabine used in conjunction with oxaliplatin in patients treated for advanced colorectal cancer (CRC). Europ J Cancer 2005;41:1542-1546.

15 Meydan N, Kundak I, Yavuzsen T, Oztop I, Barutka S, Yilmaz U, Alakavuklaar M: Cardiotoxicity of de Gramont's regimen: incidence, clinical characteristics and long-term follow-up. Jpn J Clin Oncol 2005;35:265-270. 